\title{
104 - Awareness of Dementia and Coping to Preserve Quality of Life: A Five-Year Longitudinal
} Narrative Study

\author{
Thorsen, K. ${ }^{1,2}$ Dourado, M. C. N. ${ }^{4}$, and Johannessen, A. ${ }^{1,3}$ \\ ${ }^{1}$ Vestfold Hospital Trust, Norwegian National Advisory Unit on Ageing and Health, Norway \\ ${ }^{2}$ Norwegian Social Research (NOVA), Oslo Metropolitan University, Norway \\ ${ }^{3}$ University of South-Eastern Norway \\ ${ }^{3}$ Center for Alzheimer's Disease and Related Disorders, Institute of Psychiatry, Universidade Federal do \\ Rio de Janeiro, Rio de Janeiro, RJ, Brazil
}

\begin{abstract}
Background: Awareness of dementia is examined in different scientific fields as significant for assessment of diagnosis, and for treatment and adaptation to the disease. There are very few longitudinal studies of individual experiences of awareness among people with dementia, related to quality of life.
\end{abstract}

Aim: To examine how younger people ( $<65$ years) with dementia (YOD) express awareness of the dementia and how, over time, they seem to handle awareness as a strategy to preserve quality of life.

Method: A longitudinal qualitative study with individuals with YOD was performed with interviews every six months over five years for a maximum of ten interviews. The interviews were analysed by modified grounded theory.

Findings: Awareness is a complex, multidimensional concept. Awareness of dementia is predisposed by personality, life history and established coping styles. The main coping styles - live in the moment, ignore the dementia, and make the best of it - seem to be rather consistent throughout the progression of the disease. Transitions in life situation, such as moving to a nursing home, may change the individual's awareness of dementia.

Conclusion: Unawareness of dementia may have an important adaptive function to preserve quality of life. To increase awareness must be approached with reflexivity and the utmost sensitivity.

Keywords: awareness; coping; dementia; early onset dementia; longitudinal; qualitative narrative study; quality of life; young onset dementia

Services for people with Young Onset Dementia and satisfaction with care: Findings from a national survey (the Angela project) Jan R Oyebode ${ }^{1}$, Vasileios Stamou ${ }^{1}$, Jenny LaFontaine ${ }^{1}$, Heather Gage ${ }^{2}$, Bridget Jones ${ }^{2}$, Jackie Parkes ${ }^{4}$, Mary O'Malley ${ }^{4}$, Janet Carter ${ }^{3}$.

${ }^{1}$ Centre for Applied Dementia Studies, University of Bradford, UK

${ }^{2}$ Surrey Health Economics Centre, University of Surrey, UK

${ }^{3}$ Division of Psychiatry, University College London and North East London NHS Foundation Trust

${ }^{4}$ University of Northampton

Background: It is widely recognised that specialist multidisciplinary services are required to meet the needs of people with young onset dementia for assessment, diagnosis and care. Available evidence from UK health care professionals suggests that numerous gaps exist in the provision of care; however, the detail around services used, service satisfaction and service costs is not known. 